\title{
Modulation of the central carbon metabolism of Corynebacterium glutamicum improves malonyl-CoA availability and increases plant polyphenol synthesis
}

\author{
Lars Milke $^{1 *} \mid$ Patrícia Ferreira $^{2,3 *}$ | Nicolai Kallscheuer ${ }^{1}$ (1) Adelaide Braga ${ }^{2,3} \mid$ \\ Michael Vogt $^{1}$ | Jannick Kappelmann ${ }^{1}$ | Joana Oliveira ${ }^{2,3}$ | Ana Rita Silva ${ }^{2,3}$ \\ Isabel Rocha ${ }^{2,3,4}$ | Michael Bott ${ }^{1}$ | Stephan Noack ${ }^{1}$ (0) | Nuno Faria ${ }^{2,3}$ |
}

Jan Marienhagen ${ }^{1}$

\begin{abstract}
${ }^{1}$ Institute of Bio- and Geosciences, IBG-1: Biotechnology, Forschungszentrum Jülich, Jülich, Germany

${ }^{2}$ Biotempo, Guimarães, Portugal

${ }^{3}$ Centre of Biological Engineering, University of Minho-Campus de Gualtar, Braga, Portugal

${ }^{4}$ Instituto de Tecnologia Química e Biológica, Universidade Nova de Lisboa, Oeiras, Portugal

\section{Correspondence}

Jan Marienhagen, Institute of Bio- and Geosciences, IBG-1: Biotechnology,

Forschungszentrum Jülich, Jülich, Germany.

Email: j.marienhagen@fz-juelich.de

Funding information

European Union Framework Program 7, Grant/Award Number: FP7- 613793;

Portuguese Foundation for Science and Technology, Grant/Award Numbers: NORTE01, POCI-01-0145-FEDER-006684, UID/BIO/ 04469
\end{abstract}

\begin{abstract}
In recent years microorganisms have been engineered towards synthesizing interesting plant polyphenols such as flavonoids and stilbenes from glucose. Currently, the low endogenous supply of malonyl-CoA, indispensable for plant polyphenol synthesis, impedes high product titers. Usually, limited malonyl-CoA availability during plant polyphenol production is avoided by supplementing fatty acid synthesis-inhibiting antibiotics such as cerulenin, which are known to increase the intracellular malonylCoA pool as a side effect. Motivated by the goal of microbial polyphenol synthesis being independent of such expensive additives, we used rational metabolic engineering approaches to modulate regulation of fatty acid synthesis and flux into the tricarboxylic acid cycle (TCA cycle) in Corynebacterium glutamicum strains capable of flavonoid and stilbene synthesis. Initial experiments showed that sole overexpression of genes coding for the native malonyl-CoA-forming acetyl-CoA carboxylase is not sufficient for increasing polyphenol production in C. glutamicum. Hence, the intracellular acetyl-CoA availability was also increased by reducing the flux into the TCA cycle through reduction of citrate synthase activity. In defined cultivation medium, the constructed $C$. glutamicum strains accumulated $24 \mathrm{mg} \cdot \mathrm{L}^{-1}(0.088 \mathrm{mM})$ naringenin or $112 \mathrm{mg} \cdot \mathrm{L}^{-1}$ $(0.49 \mathrm{mM})$ resveratrol from glucose without supplementation of phenylpropanoid precursor molecules or any inhibitors of fatty acid synthesis.
\end{abstract}

\section{KEYWORDS}

citrate synthase, Corynebacterium glutamicum, malonyl-CoA, naringenin, polyphenols, resveratrol

\section{1 | INTRODUCTION}

Across all domains of life, malonyl-CoA serves as building block for chain elongation steps during fatty acid biosynthesis. Beyond that, mostly in bacteria, fungi, and plants, malonyl-CoA is also required for

${ }^{*}$ These authors contributed equally to this work. the synthesis of polyketides, a large class of secondary metabolites with a multitude of functions (Han et al., 2000; van SummerenWesenhagen \& Marienhagen, 2015; Zabala, Braña, Salas, \& Méndez, 2015). In plants, polyphenols such as stilbenes and chalcones are produced from a phenylpropanoyl-CoA starter molecule (derived from the aromatic amino acids L-phenylalanine or L-tyrosine) and three molecules of malonyl-CoA (Figure 1). In this context, plant-derived 


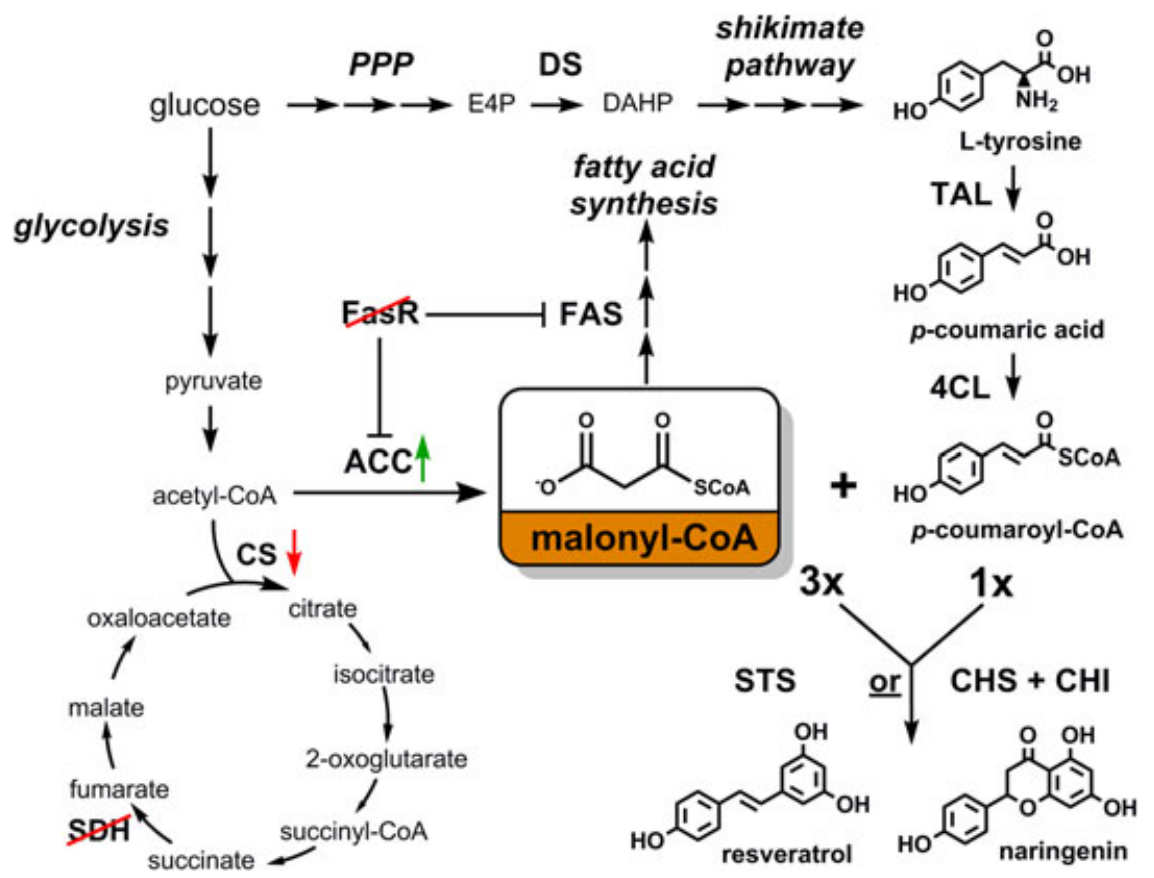

FIGURE 1 Metabolic pathways involved in the synthesis of the polyphenol precursors $p$-coumaroyl-CoA and malonyl-CoA. $p$-CoumaroylCoA and malonyl-CoA are key precursor molecules for the synthesis of the plant polyphenols naringenin and resveratrol. $p$-Coumaroyl-CoA is the CoA thioester of $p$-coumaric acid, which in turn is obtained from L-tyrosine by nonoxidative deamination. Malonyl-CoA is synthesized by carboxylation of acetyl-CoA. Metabolic pathways involved in the synthesis or consumption of the mentioned polyphenol precursors include the pentose phosphate pathway (supply of the shikimate pathways substrate erythrose 4-phosphate), the shikimate pathway (synthesis of L-tyrosine), glycolysis (pyruvate synthesis), and the TCA cycle (oxidation of acetyl-CoA). In the course of this study, several genetic modifications were introduced in to $C$. glutamicum and evaluated for their effect on polyphenol synthesis. 4CL: 4-coumarate: CoA ligase; ACC: acetyl-CoA carboxylase; CHI: chalcone isomerase; CHS: chalcone synthase; CS: citrate synthase; DAHP: 3-deoxy-D-arabino-heptulosonic acid; DS: 3-deoxyD-arabino-heptulosonic acid 7-phosphate synthase; E4P: erythrose 4-phosphate; FAS: fatty acid synthase; FasR: repressor of accBC-, accD1-, fas-IA- and fas-IB-expression; PPP: pentose phosphate pathway; SDH: succinate dehydrogenase; STS: stilbene synthase; TAL: tyrosine ammonia lyase. Abolished enzymes/transcriptional regulators: crossed out; increased enzyme activity: green arrow upwards, reduced enzyme activity: red arrow downwards [Color figure can be viewed at wileyonlinelibrary.com]

Type III polyketide synthases, either stilbene synthases (STS) or chalcone synthases (CHS), catalyze the malonyl-CoA-dependent chain elongation reaction of the phenylpropanoyl-CoA starter unit and the subsequent formation of a second aromatic ring (Ferrer, Jez, Bowman, Dixon, \& Noel, 1999). Stilbenes act as phytoalexins in plants, whereas chalcones are precursor molecules of more than 5,000 flavonoids known today (Ross \& Kasum, 2002). In general, polyphenols are of commercial interest because of the manifold of health-promoting effects including antioxidative, antibiotic, anti-inflammatory, antiallergic, and anticancer activities (Daglia, 2012).

Unfortunately, plants produce polyphenols only at very low rates and the overall polyphenol content is always subject to incalculable seasonal and climatic variations. Microorganisms, genetically engineered for plant polyphenol production, represent a promising alternative for the production of these valuable compounds (Milke, Aschenbrenner, Marienhagen, \& Kallscheuer, 2018). However, tight regulation of the endogenous malonyl-CoA synthesis with the aim to maintain only low levels of this fatty acid precursor in microorganisms, turned out to be the decisive bottleneck during microbial polyphenol production utilizing Escherichia coli and Saccharomyces cerevisiae (Kim \& Ahn, 2014; Leonard, Lim, Saw, \& Koffas, 2007; M. Li, Schneider,
Kristensen, Borodina, \& Nielsen, 2016; Lim, Fowler, Hueller, Schaffer, \& Koffas, 2011; Yang, Lin, Li, Linhardt, \& Yan, 2015). Hitherto, metabolic engineering of the microbial central carbon metabolism focused on increasing the metabolic flux towards malonyl-CoA formation (Zha, Rubin-Pitel, Shao, \& Zhao, 2009). The ATP-dependent carboxylation of acetyl-CoA, catalyzed by acetyl-CoA carboxylases (ACC) compromised up to four different subunits is the sole natural source of malonyl-CoA (S.-J. Li \& Cronan, 1992). To this end, genes coding for ACC subunits from various sources were heterologously expressed for increasing the intracellular malonyl-CoA availability during plant polyphenol production; a strategy, which was not always successful (Katsuyama, Funa, Miyahisa, \& Horinouchi, 2007; Miyahisa et al., 2005; van SummerenWesenhagen \& Marienhagen, 2015; Zha et al., 2009). Interestingly, in the context of these efforts, the ACC of Corynebacterium glutamicum has often been exploited in various species as this enzyme consists only of two instead of four different subunits (Gande et al., 2007). Expression of heterologous acc genes was often combined with deletion or downregulation of native genes coding for enzymes involved in competing pathways, for example, for acetate or ethanol formation from acetyl-CoA (Zha et al., 2009). However, primary fate of acetyl-CoA is its oxidation in the tricarboxylic acid cycle (TCA cycle). 
Here, deletion of the sdhCDAB operon coding for the succinate dehydrogenase complex of the TCA cycle of $E$. coli resulted in increased production of chalcone-derived (2S)-flavanones with this organism (Fowler, Gikandi, \& Koffas, 2009). A substantial increase in polyphenol titers in engineered microbial production strains can also be achieved by the addition of the antibiotic cerulenin (Leonard et al., 2008). Cerulenin acts as a nonselective, irreversibly-inhibiting agent on fatty acid synthases by covalent binding to the active site's cysteine and occupation of the $\beta$-ketoacyl-ACP synthase domain (Johansson et al., 2008; Price et al., 2000). This blocks fatty acid synthesis as the major "competing" pathway for malonyl-CoA consumption and thus increases the microbial production of polyphenols (Lim et al., 2011). Unfortunately, fatty acid biosynthesis-inhibiting agents such as cerulenin are very expensive and usually inhibit microbial growth as a result of rapid fatty acid depletion. Therefore, use of such antibiotics is not an option for larger-scale microbial polyphenol production.

C. glutamicum is already used for decades for the multimillion tonscale biotechnological production of amino acids and has been recently engineered towards producing plant polyphenols such as stilbenes, (2S)-flavanones, flavonols, and flavanonols, either from supplemented precursors or directly from glucose using natural or nonnatural pathways (Kallscheuer, Vogt, Bott, \& Marienhagen, 2017; Kallscheuer, Vogt, \& Marienhagen, 2016; Kallscheuer, Vogt, Stenzel et al., 2016). Similar to other microorganisms engineered for polyphenol production, low availability of malonyl-CoA also prohibits synthesis of higher polyphenol concentrations in C. glutamicum (Kallscheuer, Vogt, Stenzel et al., 2016).

We here addressed this challenge and followed several rational metabolic engineering strategies to modulate the central carbon metabolism of $C$. glutamicum with the aim to increase the intracellular malonyl-CoA availability for microbial plant polyphenol synthesis.

\section{2 | MATERIAL AND METHODS}

\section{1 | Bacterial strains, plasmids, media, and growth conditions}

All bacterial strains and plasmids used in this study along with their respective relevant characteristics are listed in Table 1 . C. glutamicum strains were routinely cultivated aerobically at $30^{\circ} \mathrm{C}$ in brain heart infusion (BHI) medium (Difco Laboratories, Detroit, MI) or defined CGXII medium with glucose as sole carbon and energy source (Keilhauer, Eggeling, \& Sahm, 1993). E. coli DH5 $\alpha$ was used for plasmid constructions and was cultivated in LB medium (Bertani, $1951)$ at $37^{\circ} \mathrm{C}$. Where appropriate, kanamycin $\left(50 \mu \mathrm{g} \cdot \mathrm{ml}^{-1}\right.$ for E. coli or $25 \mu \mathrm{g} \cdot \mathrm{ml}^{-1}$ for $C$. glutamicum) or spectinomycin $\left(100 \mu \mathrm{g} \cdot \mathrm{ml}^{-1}\right.$ for E. coli and C. glutamicum) was added to the medium. Bacterial growth was followed by measuring the optical density at $600 \mathrm{~nm}\left(\mathrm{OD}_{600}\right)$.

For cultivation of $C$. glutamicum, a single colony was picked from an agar plate and grown for six-eight hours in a test tube with $5 \mathrm{ml}$ $\mathrm{BHI}$ medium on a rotary shaker at $170 \mathrm{rpm}$ (first preculture). This culture was used to inoculate $50 \mathrm{ml}$ defined CGXII medium with $4 \%$ glucose in $500 \mathrm{ml}$ baffled Erlenmeyer flasks (second preculture). The cell suspensions were cultivated overnight on a rotary shaker at $130 \mathrm{rpm}$. The main culture was inoculated to an $\mathrm{OD}_{600}$ of 5 in CGXII medium with $4 \%$ glucose. For optimizing the production of the (2S)flavanone naringenin and the stilbene resveratrol, $p$-coumaric acid (solved in dimethyl sulfoxide) was added to a final concentration of $5 \mathrm{mM}$. Heterologous gene expression was induced one hour after inoculation of the main culture using $1 \mathrm{mM}$ isopropyl $\beta$-D-thiogalactopyranoside (IPTG). Where indicated, $25 \mu \mathrm{M}$ cerulenin was added to the main culture at an $\mathrm{OD}_{600}$ of $18-20.1 \mathrm{ml}$ of the culture broth was collected at defined time points and stored at $-20^{\circ} \mathrm{C}$ until extraction.

\section{2 | Plasmid and strain construction}

Standard protocols of molecular cloning, such as polymerase chain reaction (PCR), DNA restriction, and ligation were carried out for recombinant DNA work (Sambrook \& Russell, 2001). Techniques specific for C. glutamicum, for example, electroporation for the transformation of strains, were done as described previously (Eggeling \& Bott, 2005). All enzymes were obtained from Thermo Fisher Scientific (Schwerte, Germany). Codon-optimized synthetic genes for C. glutamicum ATCC13032 were obtained from Life Technologies (Darmstadt, Germany). Genes were amplified by PCR from genomic DNA or synthetic genes as a template using primers containing unique restriction sites (Table 2). PCR products were used for cloning of genes into plasmid vectors using the introduced restrictions site. For plasmids containing more than one insert, genes were cloned sequentially. In-frame gene deletions or integration of DNA into the genome of $C$. glutamicum were performed using the pK19mobsacB system (Schäfer et al., 1994) by a two-step homologous recombination method described previously (Niebisch \& Bott, 2001). All constructed plasmids were finally verified by DNA sequencing at Eurofins MWG Operon (Ebersberg, Germany).

\section{3 | Bioreactor cultivations of polyphenol-producing $C$. glutamicum strains}

All bioreactor experiments were performed in a $2 \mathrm{~L}$ bioreactor (Eppendorf, Hamburg, Germany) with $1 \mathrm{~L}$ of working volume. CGXII medium supplemented with $4 \%$ glucose but without urea and without 3-morpholino propanesulfonic acid (MOPS) was used in all bioreactor experiments. The $\mathrm{pH}$ was maintained at 7.0 by automatic addition of either $\mathrm{HCl}(3 \mathrm{M})$ or $\mathrm{NaOH}(2 \mathrm{M}$ ). Dissolved oxygen was kept above $30 \%$ saturation by feedback control of the stirring speed from $650 \mathrm{rpm}$ until a maximum of 1,200 rpm and an air-flow rate of $1 \mathrm{vvm}$ (volume air per volume medium and minute). The foam was disrupted by addition of antifoaming agent Antifoam 204 (Sigma-Aldrich, Schnelldorf, Germany). Bacterial growth was followed by measuring the $\mathrm{OD}_{600}$ (Synergy HT Multi-Detection Microplate Reader; BioTek Instruments, Winooski, VT) and obtained values were converted to biomass dry weight using a calibration curve. Heterologous gene expression was induced at biomass concentrations ranging from 0.9 to $1.4 \mathrm{~g} \cdot \mathrm{L}^{-1}$ using $1 \mathrm{mM}$ IPTG. One milliliter of the culture broth was collected at defined time points and stored at $-20^{\circ} \mathrm{C}$ until further processing. 
TABLE 1 Strains and plasmids used in this study

\begin{tabular}{|c|c|c|}
\hline Strain or plasmid & Characteristics & Source or reference \\
\hline \multicolumn{3}{|l|}{ Strains } \\
\hline \multicolumn{3}{|l|}{ C. glutamicum } \\
\hline DelAro ${ }^{4}-4 c l_{P c}$ & $\begin{array}{l}\text { C. glutamicum DelAro }{ }^{4} \text { derivative with a chromosomally encoded } \\
4 c l_{P c} \text { gene coding for } 4 \text {-coumarate: CoA ligase under control of the } \\
\left.\text { T7 promoter ( } \Delta \text { cg0344-47:: } \mathrm{P}_{\mathrm{T} 7}-4 c l_{P C}\right)\end{array}$ & $\begin{array}{l}\text { Kallscheuer, Vogt, Stenzel } \\
\text { et al. (2016) }\end{array}$ \\
\hline Nar1 & DelAro ${ }^{4}-4 c l_{P C}$ strain harboring pMKEx2_chs $s_{P h} c h i_{P h}$ & This study \\
\hline Nar1 $\Delta$ fasR & Nar1 derivative with in-frame deletion of fasR (cg2737) & This study \\
\hline Nar1 $\triangle s d h C A B$ & Nar1 derivative with in-frame deletion of $s d h C A B(\operatorname{cg} 0445-47)$ & This study \\
\hline Nar1 $\Delta$ fasR $\triangle s d h C A B$ & Nar1 derivative with an in-frame deletion of fasR and $s d h C A B$ & This study \\
\hline Nar1_A16 & $\begin{array}{l}\text { Nar1 derivative with the replacement of the native gltA promotor } \\
\text { with the dapA promotor variant A16 }\end{array}$ & This study \\
\hline Nar1_L1 & $\begin{array}{l}\text { Nar1 derivative with the replacement of the native gltA promotor } \\
\text { with the dapA promotor variant } \mathrm{L} 1\end{array}$ & This study \\
\hline Nar1_C7 & $\begin{array}{l}\text { Nar1 derivative with the replacement of the native gltA promotor } \\
\text { with the dapA promotor variant } \mathrm{C7}\end{array}$ & This study \\
\hline Nar1_C7 $\Delta$ fasR & Nar1_C7 derivative with an in-frame deletion of fas $R$ & This study \\
\hline Res1_C7 & $\begin{array}{l}\text { DelAro }{ }^{4}-4 c l_{P C} \text { strain harboring pMKEx2_sts }{ }_{A h} 4 c l_{P C} \text { and replacement } \\
\text { of the native gItA promotor with the dapA promotor variant } C 7\end{array}$ & This study \\
\hline \multicolumn{3}{|l|}{ E. coli } \\
\hline $\mathrm{DH} 5 \alpha$ & $\begin{array}{l}\mathrm{F}^{-} \text {Ф80lacZsM15 } \Delta(\text { (lacZYA-argF) U169 recA1 endA1 hsdR17 } \\
\left(\mathrm{r}_{\mathrm{k}}^{-}, \mathrm{m}_{\mathrm{k}}^{+}\right) \text {phoA supE44 thi-1 gyrA96 relA1 } \lambda^{-}\end{array}$ & Invitrogen (Karlsruhe, Germany) \\
\hline \multicolumn{3}{|l|}{ Plasmids } \\
\hline pK19mobsacB & $\begin{array}{l}\mathrm{kan}^{\mathrm{r}} ; \text { vector for allelic exchange in C. glutamicum (pK18, ori } \mathrm{V}_{E c}, \mathrm{sacB} \text {, } \\
\text { lacZ } \alpha \text { ) }\end{array}$ & Schäfer et al. (1994) \\
\hline pK19mobsacB $\Delta$ sdhCAB & Vector for in-frame deletion of $s d h C A B$ & This study \\
\hline $\mathrm{pK} 19 \mathrm{mobsacB} \Delta$ fasR & Vector for in-frame deletion of fas $R$ & $\begin{array}{l}\text { Nickel, Irzik, Van Ooyen, } \\
\text { and Eggeling (2010) }\end{array}$ \\
\hline pK19mobsacB_gltA_A16 & $\begin{array}{l}\text { Vector for exchanging the native promoter of glt } A \text { against the dapA } \\
\text { promotor variant } \mathrm{A} 16\end{array}$ & van Ooyen et al. (2012) \\
\hline pK19mobsacB_gltA_L1 & $\begin{array}{l}\text { Vector for exchanging the native promoter of gltA against the dapA } \\
\text { promotor variant L1 }\end{array}$ & van Ooyen et al. (2012) \\
\hline pK19mobsacB_gltA_C7 & $\begin{array}{l}\text { Vector for exchanging the native promotor of gltA against the dapA } \\
\text { promotor variant } C 7\end{array}$ & van Ooyen et al. (2012) \\
\hline pMKEx2 & $\begin{array}{l}\text { kan }^{\mathrm{r}} ; \text { E. coli-C. glutamicum shuttle vector (lacl, } \mathrm{P}_{\mathrm{T} 7}, \text { lacO1, pHM1519 } \\
\text { ori }_{C g} ; \mathrm{pACYC177} \text { ori }_{E C} \text { ) }\end{array}$ & $\begin{array}{l}\text { Kortmann, Kuhl, Klaffl, and } \\
\text { Bott (2015) }\end{array}$ \\
\hline pMKEx2_chs ${ }_{P h} C h i_{P h}$ & $\begin{array}{l}\mathrm{kan}^{\mathrm{r}} ; \text { pMKEx2 derivative with genes coding for chalcone synthase } \\
\text { and chalcone isomerase from Petunia } \mathrm{x} \text { hybrida (codon-optimized) }\end{array}$ & $\begin{array}{l}\text { Kallscheuer, Vogt, Stenzel } \\
\text { et al. (2016) }\end{array}$ \\
\hline pMKEx2_sts ${ }_{A h \_} 4 c l_{P C}$ & $\begin{array}{l}\mathrm{kar}^{\mathrm{r}} \text {; pMKEx2 derivative with genes coding for stilbene synthase } \\
\text { from Arachis hypogaea and 4-coumarate:CoA ligase from } \\
\text { Petroselinum crispum (codon-optimized) }\end{array}$ & $\begin{array}{l}\text { Kallscheuer, Vogt, Stenzel } \\
\text { et al. (2016) }\end{array}$ \\
\hline pEKEx3 & $\begin{array}{l}\text { spec }^{r} ; \text { E. coli-C. glutamicum shuttle vector (lacl, } \mathrm{P}_{\mathrm{tac}} \text {, lacO1, pBL1 ori } \mathrm{Cg}_{\mathrm{g}} \text {; } \\
\left.\text { pUC ori } \mathrm{E}_{E c}\right)\end{array}$ & Gande et al. (2007) \\
\hline pEKEx3_accBC_accD1 & $\begin{array}{l}\text { spec }^{r} ; \text { pEKEx } 3 \text { derivative containing } a c c B C \text { and } a c c D 1 \text { genes from } C \text {. } \\
\text { glutamicum }\end{array}$ & This study \\
\hline pEKEx3_aroH $\left.{ }_{E c_{-}} t\right|_{F j}$ & $\begin{array}{l}\text { spec }^{r} ; \mathrm{pEKEx} 3 \text { derivative containing native aroH from Escherichia coli } \\
\text { and a codon-optimized gene encoding tyrosine ammonia lyase } \\
\text { from Flavobacterium johnsoniae }\end{array}$ & $\begin{array}{l}\text { Kallscheuer, Vogt, Stenzel } \\
\text { et al. (2016) }\end{array}$ \\
\hline
\end{tabular}

kan $^{r}$ : kanamycin resistance, spec $^{r}$ : spectinomycin resistance 
TABLE 2 Oligonucleotides used in this study

\begin{tabular}{|lll}
\hline Primer name & Sequence $\left(\mathbf{5}^{\prime} \rightarrow \mathbf{3}^{\prime}\right)$ & Relevant site \\
\hline Sbfl-accBC-s & ACACCTGCAGGAAGGAGGACTGCTATGTCAGTCGAGACTAGGAAGATCACCAAGGTTCTTGTCGCTAAC & Sbfl \\
\hline Kpnl-accBC-as & TTCGGTACCTTACTTGATCTCGAGGAGAACAACGCCCTTG & Kpnl \\
\hline Kpnl-accD1-s & ATAGGTACCTCTAAGGAGGCGTTCGATGACCATTTCCTCACCTTTGATTGACGTC & Kpnl \\
\hline BamHl-accD1-as & CGTGGATCCTTACAGTGGCATGTTGCCGTGCTTG & BamHI \\
\hline chk-pEKEx3-s & GCAAATATTCTGAAATGAGCTGTTGACAATTAATCATC & - \\
\hline chk-pEKEx3-as & CGTTCTGATTTAATCTGTATCAGGCTGAAAATCTTCTC & - \\
\hline chk-sdhCAB-s & GATTTACATAACTTGCAGATAAGCC \\
\hline chk-sdhCAB-as & CGAGGTCAGAGGTCACAATCTC & - \\
\hline chk-fasR-s & GCGTGCCACTGCGCTACTCCTG & - \\
chk-fasR-as & CCGTCCCCGACAACATGTACGGCC \\
\hline chk-gltA-s & ATCGTTAACGATCTGACCCAACAA \\
\hline chk-gltA-as & CGTAAGCAGCCTCTGGCGGAA \\
\hline
\end{tabular}

Note. Restriction sites relevant for cloning are underlined.

\section{4 | Polyphenol extraction and LC-MS quantification}

Polyphenols (naringenin or resveratrol) were extracted from cultivation broth for subsequent LC-MS analysis by mixing $1 \mathrm{ml}$ of the culture broth with $1 \mathrm{ml}$ ethyl acetate and vigorous shaking (1,400 rpm, $10 \mathrm{~min}$, $20^{\circ} \mathrm{C}$ ) in a Thermomixer (Eppendorf). The suspension was centrifuged for $5 \mathrm{~min}$ at $13,000 \mathrm{rpm}$ and the ethyl acetate layer $(800 \mu \mathrm{l})$ was transferred to an organic solvent resistant deep-well plate (Eppendorf). After evaporation of the ethyl acetate overnight, dried extracts were resuspended in the same volume of acetonitrile and directly used for LC-MS analysis. Polyphenols were quantified using an Agilent ultrahighperformance LC (uHPLC) 1290 Infinity System coupled to a 6130 Quadrupole LC-MS System (Agilent Technologies, Waldbronn, Germany). LC separation was carried out with a Kinetex $1.7 \mu \mathrm{m}_{18}$ $100 \AA$ pore size column $(2.1 \times 50 \mathrm{~mm}$; Phenomenex, Torrance, CA $)$ at $50^{\circ} \mathrm{C}$. For elution, $0.1 \%$ acetic acid (solvent $A$ ) and acetonitrile supplemented with $0.1 \%$ acetic acid (solvent B) were applied as the mobile phases at a flow rate of $0.5 \mathrm{ml} \cdot \mathrm{min}^{-1}$. A gradient was used, where the amount of solvent $B$ was increased stepwise: minute 0-6: 10-30\%, minute 6-7: $30-50 \%$, minute 7-8: $50-100 \%$, and minute 8-8.5: $100-10 \%$. The mass spectrometer was operated in the negative electrospray ionization (ESI) mode, and data acquisition was performed in selected ion monitoring mode. Authentic naringenin and resveratrol standards were purchased from Sigma-Aldrich. Area values for $[\mathrm{M}-\mathrm{H}]^{-}$ mass signals were linear up to metabolite concentrations of at least $250 \mathrm{mg} \cdot \mathrm{L}^{-1}$. Benzoic acid (final concentration $100 \mathrm{mg} \cdot \mathrm{L}^{-1}$ ) was used as internal standard. Calibration curves were calculated based on analyte/ internal standard ratios for the obtained area values.

\section{5 | Extraction of malonyl-CoA as free acid malonate and quantification using LC-MS/MS}

Quenching, metabolite extraction, and sampling for intracellular quantification of malonyl-CoA in form of the free acid malonate were performed as described previously for phenylpropanoid CoAthioesters (Kallscheuer, Vogt, Kappelmann et al., 2016). Briefly, $5 \mathrm{ml}$ culture broth of each biological replicate were quenched in $15 \mathrm{ml}$ ice-cold $60 \% \mathrm{MeOH}$ in $\mathrm{ddH}_{2} \mathrm{O}(\mathrm{v} / \mathrm{v})$ in triplicates. After centrifugation of the quenched biomass, one part of the quenching supernatant was passed through a $0.2 \mu \mathrm{m}$ cellulose acetate filter and subsequently stored at $-20^{\circ} \mathrm{C}$ until analysis of malonate concentration. The residual quenching supernatant was carefully discarded from the pelleted biomass. The cell pellet was stored at $-80^{\circ} \mathrm{C}$ until preparation of the cell extract samples. In addition, one part of the culture broth was filtrated as described above of which $250 \mu$ l were diluted with $750 \mu \mathrm{l} 60 \% \mathrm{MeOH}$. This culture supernatant sample was used to quantify extracellular malonate, which was intentionally allowed to lyse from CoA. Cell extracts, culture supernatant and quenching supernatant samples were separated on an Agilent 1260 Infinity HPLC system (Agilent Technologies) equipped with a Sequant ZIC-pHILIC column $(2.1 \times 150 \mathrm{~mm})$ with $5 \mu \mathrm{m}$ particle size and an appropriate $20 \mathrm{~mm}$ guard column (internal diameter $2.1 \mathrm{~mm}$; Merck, Darmstadt, Germany) which were both maintained at $40^{\circ} \mathrm{C}$. For LC separation, $10 \mathrm{mM}$ ammonium acetate buffer $(\mathrm{pH} 9.2$, buffer $\mathrm{A})$ and acetonitrile (buffer $B$ ) were used. Before each injection, the column was equilibrated for $15 \mathrm{~min}$ at $90 \%$ B. After $5 \mu \mathrm{l}$ of each sample was injected, the following gradient was applied: 0 min: $90 \% \mathrm{~B}, 1 \mathrm{~min}$ : $90 \%$ B, 10 min: 70\% B, 25 min: 65\% B, 35 min: 10\% B, 45 min: 10\% B, 55 min: $10 \%$ B. The LC-eluent was coupled to an ESI-QqTOF MS (TripleTOF 6600; AB Sciex, Darmstadt, Germany) equipped with an IonDrive ion source. Data acquisition was performed using Analyst TF 1.7 (AB Sciex, Concord, ON, Canada).

For quantification, a fully ${ }^{13} \mathrm{C}$-labeled cell extract of $E$. coli was spiked with [Malonyl- ${ }^{13} \mathrm{C} 3$ ]-malonyl-CoA to attain a concentration of approximately $12.5 \mu \mathrm{M}$ (based on the molar weight of the free acid). The latter contained $\left[\mathrm{U}_{-}{ }^{13} \mathrm{C} 3\right]$-malonate as contamination (data not shown). This compound most probably originates from the

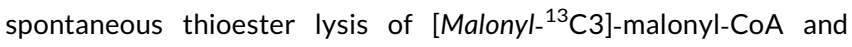
served as the internal standard for quantification of malonate. 
The internal standard solution was spiked to an equal volume of each sample and an external standard series spanning malonate concentrations from 0.01 to $100 \mu \mathrm{M}$ in $50 \% \mathrm{MeOH} / \mathrm{ddH}_{2} \mathrm{O}$ was used. A separate external standard series for malonyl-CoA was prepared analogously from the tetra lithium salt of malonyl-CoA acquired from Sigma-Aldrich.

The optimal collision energies for the most intense transitions of malonyl-CoA $(852.1>79)$ as well as malonate $(103>59)$ were -130 and $-11 \mathrm{eV}$, respectively. These energies were determined from a direct infusion of authentic standards. During the elution, these transitions as well as those of their internal standards (855.1 $>79$ and 106 > 61, respectively) were targeted in MS/MS High Sensitivity Mode using the optimal collision energy.

For both metabolites the ${ }^{12} \mathrm{C}-{ }^{13} \mathrm{C}$ isotope ratio was used for quantification. The obtained ratios for malonate were below the linear dynamic range of the calibration curve and no intracellular malonate concentrations could be calculated. Nevertheless, the intensity of the malonate signals with regard to the overall area allowed for a direct comparison of the different strains analyzed. Obtained areas for intracellular malonate were calculated as mean values with standard deviation from six samples in total per strain (two biological replicates with technical triplicates each).

\section{3 | RESULTS}

\section{1 | Modulation of the endogenous ACC activity alone does not increase polyphenol synthesis}

The parental strain C. glutamicum DelAro ${ }^{4}-4 c l_{P C}$ pMKEx2_chs ${ }_{P h} C h i_{P h}$ (designated C. glutamicum Nar1) is devoid of catabolic pathways for phenylpropanoids and benzoic acids and was previously engineered to produce naringenin from supplemented $p$-coumaric acid in presence of the fatty acid synthesis inhibitor cerulenin (Kallscheuer, Vogt, Stenzel et al., 2016). For this purpose, genes for the 4-coumarate: CoA ligase (4CL) from parsley (Petroselinum crispum) and for the CHS and the chalcone isomerase $(\mathrm{CHI})$ from Petunia $x$ hybrida are heterologously expressed. First efforts towards increasing the intracellular malonylCoA availability focused on increasing acetyl-CoA carboxylation yielding malonyl-CoA. The native $A C C$ of $C$. glutamicum is a heterodimer consisting of the two subunits $A c c B C$ ( $\alpha$-subunit) and $A c c D 1$ ( $\beta$-subunit; Gande et al., 2007). The respective genes accBC (cg0802) and accD1 (dtsR1, cg0812) were separately amplified from genomic DNA of C. glutamicum and cloned as synthetic operon under control of the tac promoter in the expression plasmid pEKEx3 yielding pEKEx3_accBC_accD1. The strain C. glutamicum Nar1 pEKEx3_accBC_accD1 was cultivated in comparison to the initial strain C. glutamicum Nar1. However, in the absence of cerulenin, no difference to the control strain with regard to naringenin titers was observable when $\operatorname{accBC}$ and accD1 were overexpressed. Both strains accumulated $2 \mathrm{mg} \cdot \mathrm{L}^{-1}$ $(0.007 \mathrm{mM})$ naringenin (data not shown). However, in the presence of $25 \mu \mathrm{M}$ cerulenin, a naringenin concentration of $26 \mathrm{mg} \cdot \mathrm{L}^{-1}(0.095 \mathrm{mM})$ could be determined for $C$. glutamicum Nar1 pEKEx3_accBC_accD1, whereas the control strain C. glutamicum Nar1 accumulated $22 \mathrm{mg} \cdot \mathrm{L}^{-1}$
$(0.081 \mathrm{mM})$ (data not shown). Noteworthy, because the presence of cerulenin inhibits fatty acid synthesis, cell growth stopped one hour after addition of this compound to the culture broth.

In C. glutamicum, the transcriptional repressor FasR controls the expression of genes coding for enzymes of the fatty acid synthesis machinery, namely the two fatty acid synthases Fas-IA (cg2743) and Fas-IB (cg0957) as well as AccBC and AccD1 (Nickel et al., 2010). In presence of increased long-chain acyl-CoA concentrations, expression of the FasR target genes is inhibited (Irzik et al., 2014). Hence, it was assumed that deletion of fasR (cg2737) would increase accBC and accD1 expression levels and thus also increases the intracellular malonyl-CoA pool. Compared with the attempted plasmid-based overexpression of $\operatorname{accBC}$ and $\operatorname{accD} 1$, this could be more beneficial with regard to a balanced expression level of both genes. Interestingly, when cultivated in CGXII medium with $4 \%$ glucose, the resulting strain C. glutamicum Nar1 $\Delta$ fasR showed a $35 \%$ reduced growth rate $\left(0.22 \mathrm{hr}^{-1}\right)$ in comparison to the reference strain C. glutamicum Nar1 $\left(0.34 \mathrm{hr}^{-1}\right)$. However, both strain strains reached the same final biomass as determined by the optical density of the cultures (Table 3 ). When comparing the polyphenol concentrations, differences between both strains were less clear as deletion of fas $R$ increased naringenin production from 2 to $4 \mathrm{mg} \cdot \mathrm{L}^{-1}(0.007-0.015 \mathrm{mM})$.

\section{2 | Reduced acetyl-CoA consumption in the central carbon metabolism increases malonyl-CoA availability}

At this stage, we speculated that an increased ACC activity would have a more pronounced effect on the malonyl-CoA pool, and ultimately on polyphenol production, when the availability of ACC substrate acetylCoA would be also increased. This can be achieved by decreasing the overall activity of the TCA cycle in C. glutamicum in which acetyl-CoA is oxidized. The citrate synthase (CS) as the pace-making enzyme of the TCA cycle, condensing acetyl-CoA and oxaloacetate to yield citrate, represents a promising target. In contrast to the closely related mycobacteria, C. glutamicum has only a single CS, which is encoded by the essential gene gltA (cg0949; van Ooyen, Noack, Bott, Reth, \& Eggeling, 2012). Hence, deletion of this gene is not a feasible strategy for reducing TCA cycle activity. Instead, expression of gltA can be downregulated by exchanging its native promoter to weaker promoter variants. This strategy was already followed previously when gltApromoter engineering in C. glutamicum improved oxaloacetate supply, ultimately leading to increased L-lysine titers (van Ooyen et al., 2012). In this particular study, eight synthetic promoter variants of the dihydrodipicolinate synthase gene dapA were constructed (Vašicová, Pátek, Nešvera, Sahm, \& Eikmanns, 1999). Three of these promoter variants, A16, L1, and C7 reducing CS activity to $32 \%, 16 \%$, and $10 \%$ compared to wild-type CS activity, respectively, were individually introduced into $C$. glutamicum Nar1. The resulting strains $C$. glutamicum Nar1_A16, C. glutamicum Nar1_L1 and C. glutamicum Nar1_C7 were further characterized with regard to growth behavior and naringenin production. C. glutamicum Nar1_A16 with 32\% residual CS activity reached the same growth rate and final biomass as the reference strain 
TABLE 3 Growth properties and naringenin titers of C. glutamicum strains engineered for naringenin production

\begin{tabular}{lcccc} 
Strain & $\begin{array}{c}\text { Growth rate } \\
\mathrm{hr}^{-1}\end{array}$ & $\begin{array}{c}\text { Final biomass } \\
\mathrm{OD}_{600}\end{array}$ & $\begin{array}{c}\text { Naringenin titer } \\
\mathrm{mg} \cdot \mathrm{L}^{-1}\end{array}$ & $\begin{array}{c}\text { Normalized naringenin titer } \\
\mathrm{mg} \cdot \mathrm{L}^{-1} \cdot \mathrm{OD}_{600}\end{array}$ \\
\hline C. glutamicum Nar1 & $0.32 \pm 0.01$ & $55.2 \pm 1.7$ & $2.1 \pm 0.5$ & 0.04 \\
\hline C. glutamicum Nar1 $\Delta$ fasR & $0.22 \pm 0.02$ & $49.6 \pm 1.5$ & $3.3 \pm 0.2$ & 0.07 \\
\hline C. glutamicum Nar1 $\Delta s$ shCAB & $0.27 \pm 0.02$ & $28.1 \pm 0.7$ & $3.8 \pm 0.2$ & 0.13 \\
\hline C. glutamicum Nar1 $\Delta$ fasR $\Delta s d h C A B$ & $0.14 \pm 0.02$ & $7.6 \pm 0.6$ & $1.9 \pm 0.9$ & 0.25 \\
\hline C. glutamicum Nar1_A16 & $0.32 \pm 0.01$ & $51.2 \pm 2.1$ & $4.5 \pm 1.2$ & 0.09 \\
\hline C. glutamicum Nar1_L1 & $0.29 \pm 0.01$ & $50.8 \pm 1.8$ & $10.3 \pm 2.4$ & 0.20 \\
\hline C. glutamicum Nar1_C7 & $0.24 \pm 0.02$ & $48.0 \pm 0.1$ & $18.5 \pm 2.5$ & 0.39 \\
\hline C. glutamicum Nar1_C7 $\Delta$ fasR & $0.19 \pm 0.02$ & $45.2 \pm 1.8$ & $12.4 \pm 1.7$ & 0.27 \\
\hline
\end{tabular}

The strains were cultivated in $50 \mathrm{ml} \mathrm{CGXII} \mathrm{medium} \mathrm{with} 4 \%$ glucose and in the presence of $5 \mathrm{mM} p$-coumaric acid. Heterologous gene expression was induced with $1 \mathrm{mM}$ IPTG. All cultivations were performed in baffled shaking flasks, and all data represent average values and standard deviations from three biological replicates.

C. glutamicum Nar1 (Table 3), but showed only a small increase of the overall polyphenol titer $\left(5 \mathrm{mg} \cdot \mathrm{L}^{-1}[0.018 \mathrm{mM}]\right.$ naringenin compared with $2 \mathrm{mg} \cdot \mathrm{L}^{-1}[0.007 \mathrm{mM}]$ obtained with C. glutamicum Nar1; Table 3). Further reduction of CS activity directly correlated with an increased naringenin production. C. glutamicum Nar1_L1 (16\% residual CS activity) and C. glutamicum Nar1_C7 (10\% residual activity) were capable of producing $12 \mathrm{mg} \cdot \mathrm{L}^{-1}(0.044 \mathrm{mM})$ and $19 \mathrm{mg} \cdot \mathrm{L}^{-1}(0.07 \mathrm{mM})$ naringenin, respectively (Table 3 ). All strains with altered gltA expression levels reached the same final biomass $\left(\mathrm{OD}_{600}\right.$ of 48-52). C. glutamicum Nar1_C7 with $10 \%$ residual CS activity was characterized by a growth rate of $0.24 \mathrm{hr}^{-1}$, which is $70 \%$ of the growth rate of the reference strain, while production of naringenin was increased 10 -fold to $19 \mathrm{mg} \cdot \mathrm{L}^{-1}$ $(0.07 \mathrm{mM})$. The strain also showed the highest naringenin titer of $0.39 \mathrm{mg} \cdot \mathrm{L}^{-1} \cdot \mathrm{OD}_{600}{ }^{-1}$ when normalized to biomass (Table 3 ).

Alternatively, we followed the strategy of deleting the $s d h C A B$ (cg0445-47) operon encoding the succinate dehydrogenase complex (SDH) in C. glutamicum. This enzyme is not essential for growth of C. glutamicum and deletion of this operon was also predicted to have a positive impact on polyphenol synthesis in a recent bioinformatics study using a genome-scale model of this bacterium (Hartmann et al., 2017). As a result of the deletion of $s d h C A B$, growth rate and final biomass of $C$. glutamicum Nar1 $\triangle s d h C A B$ were reduced by $20 \%$ and $50 \%$, respectively, in comparison to the reference strain (Table 3). Although reaching only half of the maximal biomass, $C$. glutamicum Nar1 $\triangle s d h C A B$ accumulated twice as much naringenin (C. glutamicum Nar1: $2 \mathrm{mg} \cdot \mathrm{L}^{-1}$ [0.007 mM]; C. glutamicum Nar1 $\Delta s \mathrm{shCAB:} 4 \mathrm{mg} \cdot \mathrm{L}^{-1}$ [0.015 mM]). However, additional plasmid-based expression of accBC and $\operatorname{accD} 1$ in the engineered strain $C$. glutamicum Nar1 $\triangle s d h C A B$ pEKEx3_accBC_accD1 only moderately increased the naringenin titer to $5 \mathrm{mg} \cdot \mathrm{L}^{-1}(0.018 \mathrm{mM})$ after $48 \mathrm{hr}$ of cultivation.

\section{3 | Combination of different metabolic engineering strategies}

With the aim of ascertaining whether a combination of individual strategies is suitable to further increase the production of naringenin, several genetic modifications were combined in one strain. To this end, the double deletion strain C. glutamicum Nar1 $\triangle$ fasR $\Delta s d h C A B$ was constructed to check if simultaneous deregulation of the ACC-encoding genes and reduced activity of the TCA cycle show a synergistic effect on naringenin production. Unfortunately, growth rate, final $\mathrm{OD}_{600}$ and naringenin titer of $C$. glutamicum $\triangle f a s R \Delta s d h C A B$ were drastically reduced, while the normalized naringenin titer of $0.25 \mathrm{mg} \cdot \mathrm{L}^{-1} \cdot \mathrm{OD}_{600}{ }^{-1}$ was higher compared with the parental strain C. glutamicum Nar1 (Table 3). The gene fas $R$ was also deleted in the best-performing strain $C$. glutamicum Nar1_C7, resulting in a growth rate reduction from 0.24 (C. glutamicum Nar1_C7) to $0.19 \mathrm{hr}^{-1}$ (C. glutamicum Nar1_C7 $\Delta$ fasR). Unfortunately, also in this strain the naringenin production was negatively affected. C. glutamicum Nar1_C7 $\triangle$ fasR accumulated $40 \%$ less naringenin (12 $\mathrm{mg} \cdot \mathrm{L}^{-1}$ [0.044 mM]) compared with C. glutamicum Nar1_C7, whereas final biomass concentrations $\left(\mathrm{OD}_{600}\right.$ of $45-48$ ) were similar (Table 3$)$. In contrast, plasmid-based expression of the ACC-encoding genes in the constructed strain C. glutamicum Nar1_C7 pEKEx3_accBC_accD1 increased the naringenin production by $40 \%$ to $26 \mathrm{mg} \cdot \mathrm{L}^{-1}(0.095 \mathrm{mM})$ compared with C. glutamicum Nar1_C7.

\subsection{Quantification of the intracellular malonyl- CoA pool by LC-MS/MS analysis}

Hitherto, changes in the intracellular malonyl-CoA levels in the constructed strains could be only evaluated indirectly by determination of the naringenin titer. To confirm that the constructed strains indeed generate higher amounts of malonyl-CoA, we evaluated several reported methods for intracellular malonyl-CoA extraction and quantification. However, in case of $C$. glutamicum these did either not work at all or delivered unreliable results. As a consequence, we established a method for extraction and LC-MS/MS quantification of the intracellular malonyl-CoA pools in the engineered $C$. glutamicum variants, which is based on a method we used earlier for quantifying phenylpropanoid-CoA thioesters (Kallscheuer, Vogt, Kappelmann et al., 2016). On the basis of our calibration curve for quantification, we estimate the limit of detection to be $0.1 \mu \mathrm{M}$. Given this sensitivity, we 


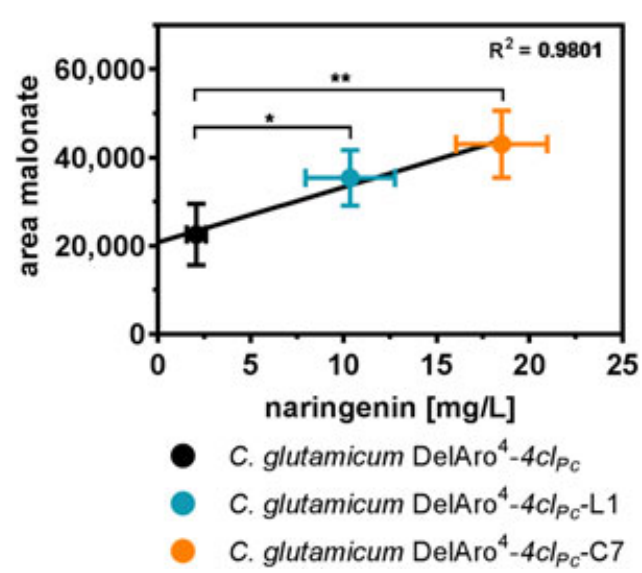

FIGURE 2 LC-MS/MS analysis of intracellular malonate concentrations. Areas for malonate acquired by LC-MS/MS analysis of cytoplasmatic extracts are plotted against the determined naringenin concentration during cultivation of the respective strains. The plotted naringenin concentrations represent mean values with standard deviations from biological triplicates, whereas the obtained malonate areas represent mean values with standard deviations from biological duplicates with three technical replicates each (six samples in total). Asterisks represent the level of significance of an unpaired two-tailed $t$ test $(p<0.05)$ [Color figure can be viewed at wileyonlinelibrary.com]

could not detect any malonyl-CoA directly in cell extracts. Interestingly, larger amounts of malonate were detectable. This acid was also present in authentic malonyl-CoA standards, due to spontaneous degradation. To the best of our knowledge, C. glutamicum is not capable of synthesizing or degrading malonate. Therefore, we expect that all malonate detectable in the analyzed samples, solely originates from such spontaneous hydrolysis of malonyl-CoA, and we also assume that higher malonate concentrations are a reflection of intracellular malonyl-CoA levels.

For all experiments, strains without the plasmid pMKEx2_chs ${ }_{P h} C h i_{P h}$ were used as any CHS activity would consume malonyl-CoA, thereby distorting the metabolite quantification (Figure 1). Due to the laborious extraction method of intracellular metabolites, only C. glutamicum strains L1 and C7 with reduced CS activity, in which corresponding naringenin titers indicated the biggest improvement in malonyl-CoA supply were compared with the reference strain C. glutamicum DelAro ${ }^{4}-4{ }_{P c}$. Because the obtained ${ }^{12} \mathrm{C}-{ }^{13} \mathrm{C}$ isotope ratios for the $C$. glutamicum DelAro ${ }^{4}-4 \mathrm{Cl}_{P C}$ and $\mathrm{L} 1$ strain were below the limit of quantification, no intracellular malonate concentration could be calculated. Subsequently, the integrated areas of the ${ }^{12} \mathrm{C}$-malonate signal of the extracted samples were adduced to allow for the comparison of all strains. The integrated areas of the malonate signals were plotted against the naringenin titers of the tested C. glutamicum strains (Figure 2). For the parental strain C. glutamicum DelAro ${ }^{4}-4{ }_{P C}$ the measured area was $22,566 \pm 6,966$, whereas malonate signals for the engineered $C$. glutamicum strains L1 and C7 were determined to be 35,426 $\pm 6,337$ (1.6-fold increase) and $43,065 \pm 757$ (1.9-fold increase), respectively. A strong linear relationship between naringenin titers and malonate areas could be observed, indicating that the developed methods for extraction and LC-MS/MS analysis of the cytoplasmic malonyl-CoA and malonate indeed reflect the intracellular concentrations of these important polyphenol precursor molecules.

\section{5 | Polyphenol production from glucose and upscaling}

The strain C. glutamicum Nar1_C7 was chosen for bioreactor cultivations as this strain accumulated the highest naringenin concentrations during shake flask cultivations. During strain engineering for improved malonyl-CoA availability, the naringenin precursor p-coumaric acid was always supplemented to exclude any possible limitation of the CHS substrate $p$-coumaroyl-CoA. However, for largescale fermentations, microbial naringenin production with $C$. glutamicum would be much more economical when starting from cheap glucose. We demonstrated earlier that the plasmid-borne heterologous expression of two additional genes is sufficient for enabling naringenin synthesis from glucose in C. glutamicum (Kallscheuer, Vogt, Stenzel et al., 2016). These genes include aroH from $E$. coli and tal from Flavobacterium johnsoniae coding for 3-deoxy-D-arabinoheptulosonate 7-phosphate synthase (the first enzyme of the shikimate pathway) and tyrosine ammonia lyase (TAL), respectively. Expression of aroH allows for the production of moderate amounts of L-tyrosine, as $\mathrm{AroH}$ of E. coli is not feedback-inhibited by this aromatic amino acid, whereas the heterologous TAL catalyzes the subsequent nonoxidative deamination of L-tyrosine yielding $p$-coumaric acid (Kallscheuer, Vogt, Stenzel et al., 2016).

C. glutamicum Nar1_C7 pEKEx3_aroH EC_ $_{-} t_{F j}$ was cultivated in a bioreactor without supplementation of $p$-coumaric acid. Naringenin production started $3 \mathrm{hr}$ after induction of heterologous gene expression. Until $28 \mathrm{hr}$ of cultivation, the product titer increased only slowly and remained below $5 \mathrm{mg} \cdot \mathrm{L}^{-1}$ ( $0.018 \mathrm{mM}$; Figure 3a). During the transition from the late exponential growth phase to the stationary phase (hours $28-35$ ), the naringenin titer increased rapidly from 4 to $20 \mathrm{mg} \cdot \mathrm{L}^{-1}(0.015-0.073 \mathrm{mM})$. The final titer of $24 \mathrm{mg} \cdot \mathrm{L}^{-1}$ $(0.088 \mathrm{mM})$ was reached at the end of the cultivation, $52 \mathrm{hr}$ after inoculation (Figure 3a). In shaking flasks with the same defined medium (only MOPS-buffered), a maximum growth rate of only $0.08 \mathrm{hr}^{-1}$ and a naringenin titer of $1.7 \mathrm{mg} \cdot \mathrm{L}^{-1}(0.006 \mathrm{mM})$ could be determined (data not shown).

In principle, flavonoids and stilbenes share the same metabolic route, which is also reflected in the requirement of three mol malonyl-CoA $/ \mathrm{mol}$ product for both compound classes (Figure 1). With the aim to demonstrate that the improvements with regard to intracellular malonyl-CoA availability are transferable from flavonoid synthesis to stilbene synthesis, additional bioreactor cultivations were performed. In this context, C. glutamicum Res1_C7 pEKEx3_ar$\mathrm{oH}_{E C_{-}} t a_{F j}$ as the engineered variant with the largest intracellular malonyl-CoA pool was constructed using the plasmid pMKEx2_st$s_{A h_{-}} 4 c l_{P C}$ conferring the capability to produce the stilbene resveratrol (Kallscheuer, Vogt, Stenzel et al., 2016). In performed bioreactor cultivations the strain reached a final biomass concentration of $8.7 \mathrm{~g} \cdot \mathrm{L}^{-1}$ and exhibited a specific growth rate of $0.20 \mathrm{hr}^{-1}$ (Figure $3 \mathrm{~b}$ ). Resveratrol synthesis set in $3 \mathrm{hr}$ after induction of heterologous gene 
(a)

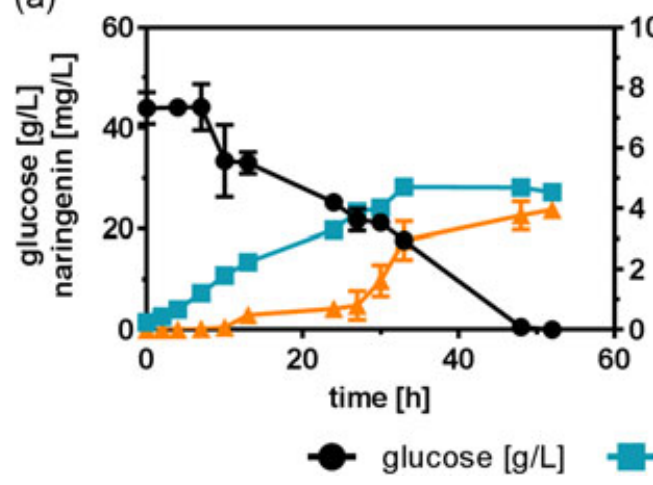

(b)

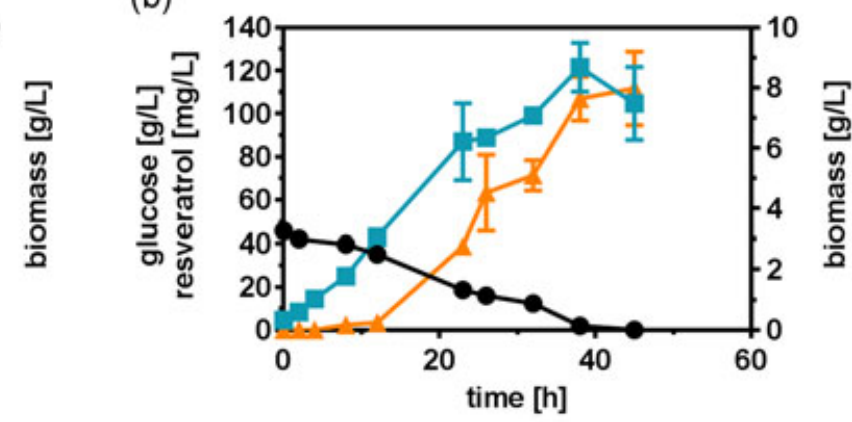

biomass $[g / L]$

polyphenol $[\mathrm{mg} / \mathrm{L}]$

FIGURE 3 Microbial production of naringenin and resveratrol from glucose during bioreactor cultivations with engineered C. glutamicum strains. (a) Naringenin production from glucose: concentration of glucose and biomass and naringenin titers during batch cultivation of C. glutamicum Nar1_C7 pEKEx3_aroH Ec_tal $_{F j}$. (b) Resveratrol production from glucose: concentration of glucose and biomass and resveratrol titers during batch cultivation of $C$. glutamicum Res1_C7 pEKEx3_aroH $H_{E c} t_{F} I_{F j}$. The data represent average values and standard deviations from three independent experiments [Color figure can be viewed at wileyonlinelibrary.com]

expression and a final maximum resveratrol concentration of $112 \mathrm{mg} \cdot \mathrm{L}^{-1}(0.491 \mathrm{mM})$ could be determined (Figure $\left.3 \mathrm{~b}\right)$. These experiments show that the improvements achieved with regard to the intracellular malonyl-CoA availability are beneficial for flavonoidas well as stilbene production with $C$. glutamicum, emphasizing the versatility of this platform organism for plant polyphenol synthesis.

\section{4 | DISCUSSION}

Currently, microbial synthesis of polyphenols with C. glutamicum, as well as with other microorganisms engineered towards producing these compounds, relies on the supplementation of cerulenin, which acts as an inhibitor of fatty acid synthesis (Kallscheuer, Vogt, Stenzel et al., 2016). Although this strategy is often successfully applied in the context of microbial synthesis of various stilbenes, (2S)-flavanones, flavones, and flavonols at lab scale, it was early recognized that any large-scale microbial polyphenol production using cerulenin would be uneconomical due to the high costs of this compound (Milke et al., 2018).

The expression of genes coding for ACCs is a reasonable strategy to increase the endogenous pool of malonyl-CoA, which is the ratelimiting metabolite for polyketide-derived compounds in engineered bacteria. Streptomyces venezuelae, an actinobacterium related to C. glutamicum, is a natural producer of the antibiotic jadomycin, which requires altogether nine molecules of malonyl-CoA per mol product (Jakeman et al., 2009). Interestingly, in the gene cluster essential for jadomycin synthesis, the gene jadJ codes for an ACC $\alpha$-subunit (Han et al., 2000). Deletion of this gene in $S$. venezuelae severely affected jadomycin production, which underlines the significance of high ACC activity for sufficient malonyl-CoA levels. In the context of engineering microorganisms for polyphenol production, the heterodimeric ACC from C. glutamicum was used several times to overcome the tight regulation of endogenous malonyl-CoA synthesis in E. coli (Miyahisa et al., 2005; van Summeren-Wesenhagen \& Marienhagen, 2015; Zha et al., 2009; Zhao, Wu, Liu, Qiao, \& Zhao, 2018). In C. glutamicum, the presence of long-chain acyl-CoA molecules or transition from the exponential phase to the stationary phase leads to downregulation of $\operatorname{accBC}$ and $a c c D 1$ expression (Irzik et al., 2014; Larisch, Nakunst, Hüser, Tauch, \& Kalinowski, 2007). In our study, both genes were put under control of the IPTG-inducible tac promoter and expressed as a bicistronic transcript with the aim to circumvent this regulation. Alternatively, the gene coding for the fatty acid repressor FasR was deleted, a strategy, which proved to be successful in the past when C. glutamicum was engineered for the overproduction of fatty acids (Takeno et al., 2013). Unfortunately, both approaches resulted only in a moderate increase of naringenin biosynthesis. The limited effect of the deletion of fas $R$ on naringenin synthesis could be explained by the previous finding that absence of the transcriptional repressor fas $R$ in C. glutamicum also relieves repression of both genes encoding for the fatty acid synthases Fas-IA and Fas-IB, which consume malonyl-CoA (Nickel et al., 2010). Presumably, this leads to increased fatty acid synthesis and does not necessarily provide more malonyl-CoA for plant polyphenol synthesis.

From the data obtained to this point, we concluded that increased expression of the ACC-encoding genes only leads to an increase in malonyl-CoA synthesis when the acetyl-CoA-level is also increased. In E. coli, deletion of the $s d h$-operon was performed to increase acetylCoA availability for polyphenol synthesis (Fowler et al., 2009). As a result, naringenin titers increased 1.5 -fold. In case of $C$. glutamicum, it has already been described that loss of SDH activity interrupting the TCA cycle results in reduced growth and accumulation of succinate and acetate up to titers of 40 and $125 \mathrm{mM}$, respectively (Litsanov, Kabus, Brocker, \& Bott, 2012). Nonetheless, an SDH-deficient C. glutamicum variant was constructed to find out how the loss of $\mathrm{SDH}$ activity impacts polyphenol synthesis. Interestingly, naringenin production increased 1.8-fold, but growth rate and overall biomass formation were drastically reduced, rendering this strain unsuitable for further engineering towards microbial polyphenol synthesis. In contrast, reduction of the endogenous CS activity turned out to be 
more beneficial as the growth of $C$. glutamicum was less negatively affected and polyphenol concentrations were increased. Reduction of the CS activity already proved to be successful during metabolic engineering of a $C$. glutamicum strain towards microbial L-lysine production (van Ooyen et al., 2012). In this study, reduction of the CS activity increased L-lysine concentration in the culture supernatant from 7.51 to $13.19 \mathrm{~g} \cdot \mathrm{L}^{-1}$. The same $C$. glutamicum strain, only differing in the genetic constitution related to product synthesis, was found to produce different naringenin $\left(24 \mathrm{mg} \cdot \mathrm{L}^{-1}\right)$ and resveratrol $\left(112 \mathrm{mg} \cdot \mathrm{L}^{-1}\right)$ concentrations from glucose, although both molecules are derived from the same precursor molecules. This observation is in consistency with a preceding study from our lab, in which synthesis of resveratrol seemed to be more efficient compared with the synthesis of naringenin (Kallscheuer, Vogt, Stenzel et al., 2016).

When comparing microbial resveratrol and naringenin production in C. glutamicum, E. coli or S. cerevisiae, either from supplemented precursors or directly from glucose, resveratrol titers generally seem to exceed the ones of naringenin independent from the utilized host or the heterologous genes used (Chouhan, Sharma, Zha, Guleria, \& Koffas, 2017; Jeandet et al., 2018; Milke et al., 2018). Similar observations have also been made for two very similar $E$. coli strains also engineered towards naringenin and resveratrol synthesis (Yang et al., 2015). This suggests that the difference in product concentrations observed might be due to the chemical reactions catalyzed by STS and $\mathrm{CHS} / \mathrm{CHI}$, respectively. We calculated the change of the Gibbs free energy for the final reaction of resveratrol and naringenin biosynthesis using the BioCYC database (Caspi et al., 2007; Caspi et al., 2015) to obtain the free energy for all metabolites (substrates and products) involved. Indeed, we found that the final step of the resveratrol biosynthesis appears to be thermodynamically more favorable compared to the last step of naringenin biosynthesis $\left(\Delta G^{0,}=-70 \mathrm{~kJ} \cdot \mathrm{mol}^{-1}\right.$ for resveratrol compared with $\Delta G^{0,}=+46 \mathrm{~kJ} \cdot \mathrm{mol}^{-1}$ for naringenin). This difference is due to an additional molecule of $\mathrm{CO}_{2}$ released during resveratrol synthesis and might help to explain this interesting observation.

Taken together, by reducing the CS activity in the parental strain C. glutamicum DelAro ${ }^{4}-4{ }_{P C}$, dependency on the costly antibiotic cerulenin for efficient polyphenol synthesis could be overcome. As demonstrated by the applicability of the best performing C. glutamicum DelAro ${ }^{4}-4{ }_{P_{C}-} C 7$ strain for both, flavonoid and resveratrol synthesis, it can be excluded that the observed improvements regarding malonyl-CoA availability are for any reason limited to naringenin synthesis only. Thus, the C. glutamicum DelAro ${ }^{4}$ $4 c l_{P C_{-}} \mathrm{C7}$ strain constructed in this study represents a promising starting point for further tailoring this bacterium towards increased microbial plant polyphenol synthesis.

\section{ACKNOWLEDGMENTS}

We would like to thank the European Union Framework Program 7 "BacHBerry" (www.bachberry.eu), Project No. FP7- 613793 for financial support and also the Portuguese Foundation for Science and Technology (FCT) under the scope of the strategic funding of UID/ BIO/04469 unit, COMPETE 2020 (POCI-01-0145-FEDER-006684) and BiotecNorte operation (NORTE-01-0145-FEDER-000004) funded by the European Regional Development Fund under the scope of Norte2020 - Programa Operacional Regional do Norte. Furthermore, we would like to thank Dr. Tino Polen for providing the plasmids to replace the gItA promoter by dapA promoter variants.

\section{CONFLICT OF INTERESTS}

The authors declare that they have no conflict of interests.

\section{ORCID}

Nicolai Kallscheuer (D) http://orcid.org/0000-0003-4925-6923

Stephan Noack (D) http://orcid.org/0000-0001-9784-3626

Jan Marienhagen (iD) http://orcid.org/0000-0001-5513-3730

\section{REFERENCES}

Bertani, G. (1951). Studies on lysogenesis I: The mode of phage liberation by lysogenic Escherichia coli. Journal of Bacteriology, 62(3), 293-300.

Caspi, R., Foerster, H., Fulcher, C. A., Kaipa, P., Krummenacker, M., Latendresse, M., ... Karp, P. D. (2007). The MetaCyc database of metabolic pathways and enzymes and the BioCyc collection of pathway/genome databases. Nucleic Acids Research, 36, D623-D631. https://doi.org/10.1093/nar/gkm900

Caspi, R., Billington, R., Ferrer, L., Foerster, H., Fulcher, C. A., Keseler, I. M., ... Karp, P. D. (2015). The MetaCyc database of metabolic pathways and enzymes and the BioCyc collection of pathway/genome databases. Nucleic Acids Research, 44(D1), D471-D480. https://doi.org/10.1093/nar/gkv1164

Chouhan, S., Sharma, K., Zha, J., Guleria, S., \& Koffas, M. A. G. (2017). Recent advances in the recombinant biosynthesis of polyphenols. Frontiers in Microbiology, 8, 2259. https://doi.org/10.3389/fmicb.2017.02259

Daglia, M. (2012). Polyphenols as antimicrobial agents. Current Opinion in Biotechnology, 23(2), 174-181. https://doi.org/10.1016/j.copbio.2011. 08.007

Eggeling, L. , \& Bott, M. (2005). Handbook of Corynebacterium glutamicum (537-564). Boca Raton, FL: CRC press. https://doi.org/10.1201/ 9781420039696

Ferrer, J. -L., Jez, J. M., Bowman, M. E., Dixon, R. A., \& Noel, J. P. (1999). Structure of chalcone synthase and the molecular basis of plant polyketide biosynthesis. Nature Structural Biology, 6(8), 775-784. https://doi.org/10.1038/11553

Fowler, Z. L., Gikandi, W. W., \& Koffas, M. A. G. (2009). Increased malonyl coenzyme $\mathrm{A}$ biosynthesis by tuning the Escherichia coli metabolic network and its application to flavanone production. Applied and Environmental Microbiology, 75(18), 5831-5839. https://doi.org/10. 1128/AEM.00270-09

Gande, R., Dover, L. G., Krumbach, K., Besra, G. S., Sahm, H., Oikawa, T., \& Eggeling, L. (2007). The two carboxylases of Corynebacterium glutamicum essential for fatty acid and mycolic acid synthesis. Journal of Bacteriology, 189(14), 5257-5264. https://doi.org/10.1128/JB.00254-07

Hartmann, A., Vila-Santa, A., Kallscheuer, N., Vogt, M., Julien-Laferrière, A., Sagot, M. -F., ... Vinga, S. (2017). OptPipe-A pipeline for optimizing metabolic engineering targets. BMC Systems Biology, 11(1), 143. https://doi.org/10.1186/s12918-017-0515-0

Irzik, K., van Ooyen, J., Gätgens, J., Krumbach, K., Bott, M., \& Eggeling, L. (2014). Acyl-CoA sensing by FasR to adjust fatty acid synthesis in Corynebacterium glutamicum. Journal of Biotechnology, 192, 96-101. https://doi.org/10.1016/j.jbiotec.2014.10.031

Jakeman, D. L., Bandi, S., Graham, C. L., Reid, T. R., Wentzell, J. R., \& Douglas, S. E. (2009). Antimicrobial activities of jadomycin B and 
structurally related analogues. Antimicrobial Agents and Chemotherapy, 53(3), 1245-1247. https://doi.org/10.1128/AAC.00801-08

Jeandet, P., Sobarzo-Sánchez, E., Clément, C., Nabavi, S. F., Habtemariam, S., Nabavi, S. M., \& Cordelier, S. (2018). Engineering stilbene metabolic pathways in microbial cells. Biotechnology Advances, 36, 2264-2283. https://doi.org/10.1016/j.biotechadv.2018.11.002

Johansson, P., Wiltschi, B., Kumari, P., Kessler, B., Vonrhein, C., Vonck, J., ... Grininger, M. (2008). Inhibition of the fungal fatty acid synthase type I multienzyme complex. Proceedings of the National Academy of Sciences, 105(35), 12803-12808. https://doi.org/10.1073/pnas. 0805827105

Kallscheuer, N., Vogt, M., Bott, M., \& Marienhagen, J. (2017). Functional expression of plant-derived O-methyltransferase, flavanone 3-hydroxylase, and flavonol synthase in Corynebacterium glutamicum for production of pterostilbene, kaempferol, and quercetin. Journal of Biotechnology, 258, 190-196. https://doi.org/10.1016/j.jbiotec.2017. 01.006

Kallscheuer, N., Vogt, M., Kappelmann, J., Krumbach, K., Noack, S., Bott, M., \& Marienhagen, J. (2016). Identification of the phd gene cluster responsible for phenylpropanoid utilization in Corynebacterium glutamicum. Applied Microbiology and Biotechnology, 100(4), 1871-1881. https://doi.org/10.1007/s00253-015-7165-1

Kallscheuer, N., Vogt, M., \& Marienhagen, J. (2016). A novel synthetic pathway enables microbial production of polyphenols independent from the endogenous aromatic amino acid metabolism. ACS Synthetic Biology, 6(3), 410-415. https://doi.org/10.1021/acssynbio.6b00291

Kallscheuer, N., Vogt, M., Stenzel, A., Gätgens, J., Bott, M., \& Marienhagen, J. (2016). Construction of a Corynebacterium glutamicum platform strain for the production of stilbenes and (2S)flavanones. Metabolic Engineering, 38, 47-55. https://doi.org/10. 1016/j.ymben.2016.06.003

Katsuyama, Y., Funa, N., Miyahisa, I., \& Horinouchi, S. (2007). Synthesis of unnatural flavonoids and stilbenes by exploiting the plant biosynthetic pathway in Escherichia coli. Chemistry \& Biology, 14(6), 613-621. https://doi.org/10.1016/j.chembiol.2007.05.004

Keilhauer, C., Eggeling, L., \& Sahm, H. (1993). Isoleucine synthesis in Corynebacterium glutamicum: Molecular analysis of the ilvB-ilvN-ilvC operon. Journal of Bacteriology, 175(17), 5595-5603. https://doi.org/ 10.1128/jb.175.17.5595-5603.1993

Kim, B. G., Lee, H., \& Ahn, J. H. (2014). Biosynthesis of pinocembrin from glucose using engineered Escherichia coli. Journal of Microbiology and Biotechnology, 24(11), 1536-1541. https://doi.org/10.4014/jmb.1406. 06011

Kortmann, M., Kuhl, V., Klaffl, S., \& Bott, M. (2015). A chromosomally encoded T7 RNA polymerase-dependent gene expression system for Corynebacterium glutamicum: Construction and comparative evaluation at the single-cell level. Microbial Biotechnology, 8(2), 253-265. https://doi.org/10.1111/1751-7915.12236

Han, L., Yang, K., Kulowski, K., Wendt-Pienkowski, E., Hutchinson, C. R., \& Vining, L. C. (2000). An acyl-coenzyme A carboxylase encoding gene associated with jadomycin biosynthesis in Streptomyces venezuelae ISP5230. Microbiology, 146(4), 903-910.

Larisch, C., Nakunst, D., Hüser, A. T., Tauch, A., \& Kalinowski, J. (2007). The alternative sigma factor SigB of Corynebacterium glutamicum modulates global gene expression during transition from exponential growth to stationary phase. BMC Genomics, 8(1), 1. https://doi.org/10. 1186/1471-2164-8-4

Leonard, E., Lim, K. -H., Saw, P. -N., \& Koffas, M. A. G. (2007). Engineering central metabolic pathways for high-level flavonoid production in Escherichia coli. Applied and Environmental Microbiology, 73(12), 3877-3886. https://doi.org/10.1128/AEM.00200-0

Leonard, E., Yan, Y., Fowler, Z. L., Li, Z., Lim, C.-G., Lim, K.-H., \& Koffas, M. A. G. (2008). Strain improvement of recombinant Escherichia coli for efficient production of plant flavonoids. Molecular Pharmaceutics, 5(2), 257-265. https://doi.org/10.1021/mp7001472

Li, M., Schneider, K., Kristensen, M., Borodina, I., \& Nielsen, J. (2016). Engineering yeast for high-level production of stilbenoid antioxidants. Scientific Reports, 6, 36827. https://doi.org/10.1038/srep36827

Li, S.-J., \& Cronan, J. E. (1992). The genes encoding the two carboxyltransferase subunits of Escherichia coli acetyl-CoA carboxylase. Journal of Biological Chemistry, 267(24), 16841-16847.

Lim, C. G., Fowler, Z. L., Hueller, T., Schaffer, S., \& Koffas, M. A. G. (2011). High-yield resveratrol production in engineered Escherichia coli. Applied and Environmental Microbiology, 77(10), 3451-3460. https:// doi.org/10.1128/AEM.02186-10

Litsanov, B., Kabus, A., Brocker, M., \& Bott, M. (2012). Efficient aerobic succinate production from glucose in minimal medium with Corynebacterium glutamicum. Microbial Biotechnology, 5(1), 116-128. https:// doi.org/10.1111/j.1751-7915.2011.00310.x

Milke, L., Aschenbrenner, J., Marienhagen, J., \& Kallscheuer, N. (2018). Production of plant-derived polyphenols in microorganisms: Current state and perspectives. Applied Microbiology and Biotechnology, 102(4), 1575-1585. https://doi.org/10.1007/s00253-018-8747-5

Miyahisa, I., Kaneko, M., Funa, N., Kawasaki, H., Kojima, H., Ohnishi, Y., \& Horinouchi, S. (2005). Efficient production of (2S)-flavanones by Escherichia coli containing an artificial biosynthetic gene cluster. Applied Microbiology and Biotechnology, 68(4), 498-504. https://doi. org/10.1007/s00253-005-1916-3

Nickel, J., Irzik, K., van Ooyen, J., \& Eggeling, L. (2010). The TetR-type transcriptional regulator FasR of Corynebacterium glutamicum controls genes of lipid synthesis during growth on acetate. Molecular Microbiology, 78(1), 253-265. https://doi.org/10.1111/j.1365-2958.2010.07337.x

Niebisch, A., \& Bott, M. (2001). Molecular analysis of the cytochrome $b c_{1}$ $a a_{3}$ branch of the Corynebacterium glutamicum respiratory chain containing an unusual diheme cytochrome $c_{1}$. Archives of Microbiology, 175(4), 282-294. https://doi.org/10.1007/s002030100262

Price, Allen C., Choi, Keum-Hwa, Heath, Richard J., Li, Zhenmei, White, Stephen W., \& Rock, Charles O. (2000). Inhibition of $\beta$-Ketoacyl-Acyl Carrier Protein Synthases by Thiolactomycin and Cerulenin. Journal of Biological Chemistry, 276(9), 6551-6559. https://doi.org/10.1074/jbc. m007101200

Ross, J. A., \& Kasum, C. M. (2002). Dietary flavonoids: Bioavailability, metabolic effects, and safety. Annual Review of Nutrition, 22(1), 19-34. https://doi.org/10.1146/annurev.nutr.22.111401.144957

Sambrook, J., \& Russell, D. (2001). Molecular cloning. Cold Spring Harbor, NY: Cold Spring Harbor Laboratory Press.

Schäfer, A., Tauch, A., Jäger, W., Kalinowski, J., Thierbach, G., \& Pühler, A. (1994). Small mobilizable multi-purpose cloning vectors derived from the Escherichia coli plasmids pK18 and pK19: Selection of defined deletions in the chromosome of Corynebacterium glutamicum. Gene, 145(1), 69-73. https://doi.org/10.1016/0378-1119(94) 90324-7

Takeno, S., Takasaki, M., Urabayashi, A., Mimura, A., Muramatsu, T., Mitsuhashi, S., \& Ikeda, M. (2013). Development of fatty acidproducing Corynebacterium glutamicum strains. Applied and Environmental Microbiology, 79(21), 6776-6783. https://doi.org/10.1128/ AEM.02003-13

van Ooyen, J., Noack, S., Bott, M., Reth, A., \& Eggeling, L. (2012). Improved L-lysine production with Corynebacterium glutamicum and systemic insight into citrate synthase flux and activity. Biotechnology and Bioengineering, 109(8), 2070-2081. https://doi.org/10. 1002/bit.24486

van Summeren-Wesenhagen, P. V., \& Marienhagen, J. (2015). Metabolic engineering of Escherichia coli for the synthesis of the plant polyphenol pinosylvin. Applied and Environmental Microbiology, 81(3), 840-849. https://doi.org/10.1128/AEM.02966-14 
Vasicová, P., Pátek, M., Nesvera, J., Sahm, H., \& Eikmanns, B. (1999). Analysis of the Corynebacterium glutamicum dapA promoter. Journal of Bacteriology, 181(19), 6188-6191.

Yang, Y., Lin, Y., Li, L., Linhardt, R. J., \& Yan, Y. (2015). Regulating malonylCoA metabolism via synthetic antisense RNAs for enhanced biosynthesis of natural products. Metabolic Engineering, 29, 217-226. https:// doi.org/10.1016/j.ymben.2015.03.018

Zabala, D., Braña, A. F., Salas, J. A., \& Méndez, C. (2015). Increasing antibiotic production yields by favoring the biosynthesis of precursor metabolites glucose-1-phosphate and/or malonyl-CoA in Streptomyces producer strains. The Journal of Antibiotics, 69, 179-182. https://doi. org/10.1038/ja.2015.104

Zha, W., Rubin-Pitel, S. B., Shao, Z., \& Zhao, H. (2009). Improving cellular malonyl-CoA level in Escherichia coli via metabolic engineering. Metabolic Engineering, 11(3), 192-198. https://doi.org/10.1016/j.ymben.2009.01.005
Zhao, Y., Wu, B. -H., Liu, Z. -N., Qiao, J., \& Zhao, G. -R. (2018). Combinatorial optimization of resveratrol production in engineered $E$. coli. Journal of Agricultural and Food Chemistry, 66, 13444-13453. https://doi.org/10.1021/acs.jafc.8b05014

How to cite this article: Milke L, Ferreira P, Kallscheuer N, et al. Modulation of the central carbon metabolism of Corynebacterium glutamicum improves malonyl-CoA availability and increases plant polyphenol synthesis. Biotechnology and Bioengineering. 2019;116:1380-1391. https://doi.org/10.1002/bit.26939 